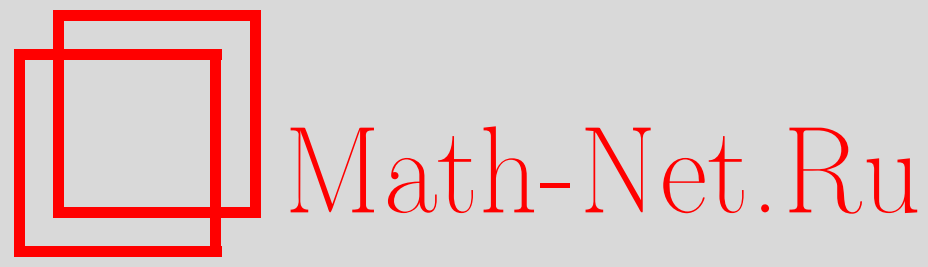

А. С. Черный, О сильной и слабой единственности для стохастических дифференциальных уравнений, Теория вероятн. и ее примен., 2001, том 46, выпуск 3, 483-497

DOI: https://doi.org/10.4213/tvp3897

Использование Общероссийского математического портала MathNet.Ru подразумевает, что вы прочитали и согласны с пользовательским соглашением

http://www . mathnet.ru/rus/agreement

Параметры загрузки:

IP : 18.209 .158 .208

26 апреля 2023 г., 02:46:55

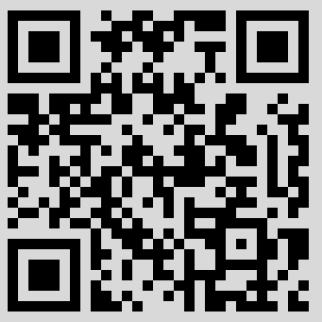




\section{О СИЛЬНОЙ И СЛАБОЙ ЕДИНСТВЕННОСТИ ДЛЯ СТОХАСТИЧЕСКИХ ДИФФЕРЕНЩИАЛЬНЫХ УРАВНЕНИЙ}

В статье доказано, что из слабой единственности для стохастического дифференциального уравнения

$$
d X_{t}^{i}=b_{t}^{i}(X) d t+\sum_{j=1}^{m} \sigma_{t}^{i j}(X) d B_{t}^{j}, \quad X_{0}^{i}=x^{i} \quad(i=1, \ldots, n)
$$

вытекает единственность совместного распределения пары $(X, B)$.

Кроме того, доказывается, что если для $(*)$ имеет место слабая единственность и существует сильное решение, то имеет место сильная единственность. Этот результат является в некотором смысле «двойственным» к теореме Ямада-Ватанабэ.

Ключевые слова и фразы: стохастические дифференциальные уравнения, слабые решения, сильные решения, слабая единственность, сильная единственность, теорема Ямада-Ватанабэ.

1. Введение. Пусть $C\left(\mathbf{R}_{+}, \mathbf{R}^{n}\right)$ - пространство непрерывных функций $\mathbf{R}_{+} \rightarrow \mathbf{R}^{n}$. Напомним, что канонический прочесс $Y=\left(Y_{t}\right)_{t \geqslant 0}$ на этом пространстве определяется по формуле

$$
Y_{t}: C\left(\mathbf{R}_{+}, \mathbf{R}^{n}\right) \ni y \longmapsto y(t) \in \mathbf{R}^{n} .
$$

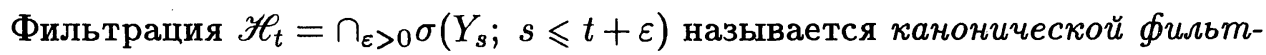
рачией на $C\left(\mathbf{R}_{+}, \mathbf{R}^{n}\right)$. Предсказуемая $\sigma$-алгебра на $C\left(\mathbf{R}_{+}, \mathbf{R}^{n}\right)$ - это $\sigma$-алгебра, порожденная непрерывными слева $\left(\mathscr{H}_{t}\right)$-согласованными процессами на $C\left(\mathbf{R}_{+}, \mathbf{R}^{n}\right)$.

B настоящей статье мы будем рассматривать многомерные стохастические дифференциальные уравнения (в дальнейшем будет использоваться сокращение СДУ) следующего вида:

$$
d X_{t}^{i}=b_{t}^{i}(X) d t+\sum_{j=1}^{m} \sigma_{t}^{i j}(X) d B_{t}^{j}, \quad X_{0}^{i}=x^{i} \quad(i=1, \ldots, n),
$$

где $n \in \mathbf{N}, m \in \mathbf{N}, x \in \mathbf{R}^{n}$, a $b, \sigma$ - предсказуемые процессы на $C\left(\mathbf{R}_{+}, \mathbf{R}^{n}\right)$, принимающие значения в $\mathbf{R}^{n}$ и $\mathbf{R}^{n \times m}$ соответственно.

\footnotetext{
${ }^{*}$ Московский государственный университет им. М. В. Ломоносова, механико-математический факультет, кафедра теории вероятностей, Воробьевы горы, 119899 Москва, Россия; e-mail: cherny@mech.math.msu.su
} 
3 а м е ч а н и е. Начальная точка $x$ фиксируется вместе с $b$ и $\sigma$. Согласно нашей терминологии, СДУ с одинаковыми $b$ и $\sigma$, но с разными начальными точками считаются различными.

О п р е д е л н и е 1.1. Решение уравнения (1.1) - это пара $(X, B)$ согласованных процессов на некотором фильтрованном вероятностном пространстве $\left(\Omega, \mathscr{F},\left(\mathscr{F}_{t}\right)_{t \geqslant 0}, \mathbf{P}\right)$ такая, что

i) $B$ является $\left(\mathscr{F}_{t}, \mathbf{P}\right)-\mathrm{BM}^{m}(0)$, т.е. $B-m$-мерное броуновское движение и $\left(\mathscr{F}_{t}, \mathbf{P}\right)$-мартингал;

ii) для любого $t \geqslant 0$

$$
\int_{0}^{t}\left(\sum_{i=1}^{n}\left|b_{s}^{i}(X)\right|+\sum_{i=1}^{n} \sum_{j=1}^{m}\left(\sigma_{s}^{i j}(X)\right)^{2}\right) d s<\infty \quad \text { Р-п.н.; }
$$

iii) для любых $t \geqslant 0, i=1, \ldots, n$

$$
X_{t}^{i}=x^{i}+\int_{0}^{t} b_{s}^{i}(X) d s+\sum_{j=1}^{m} \int_{0}^{t} \sigma_{s}^{i j}(X) d B_{s}^{j} \quad \text { Р-п.н. }
$$

3 а м е ч а н и е. В дальнейшем будем использовать векторную форму обозначений. Если $b-n$-мерный процесс, то через $\int_{0}^{t} b_{s} d s$ будет обозначаться $n$-мерный процесс, $i$-я компонента которого равна $\int_{0}^{t} b_{s}^{i} d s$ Если $M-m$-мерный локальный мартингал, а $\sigma$ - предсказуемый процесс со значениями в $\mathbf{R}^{n \times m}$, то через $\int_{0}^{t} \sigma_{s} d M_{s}$ будет обозначаться $n$-мерный процесс, $i$-я компонента которого равна $\sum_{j=1}^{m} \int_{0}^{t} \sigma_{s}^{i j} d M_{s}^{j}$. В этих обозначениях равенство (1.2) переписывается в виде

$$
X_{t}=x+\int_{0}^{t} b_{s}(X) d s+\int_{0}^{t} \sigma_{s}(X) d B_{s} \quad \text { Р-п.н. }
$$

О п р е д е л е н и е 1.2. Решение $(X, B)$ называется сильныл решением, если процесс $X$ согласован с $\left(\bar{F}_{t}^{B}\right)$, т.е. с пополненной натуральной фильтрацией процесса $B$.

3 а м е ч а н и е. Решение в смысле определения 1.1 иногда называют слабым решением. Мы же будем придерживаться просто термина решение. Однако существование такого решения будет обозначаться термином слабое существование, чтобы подчеркнуть отличие от сильного существования, т.е. существования сильного решения.

О п р е д е л е н и 1.3. Для уравнения (1.1) имеет место сла бая единственность, если для любых решений $(X, B)$ и $(\widetilde{X}, \widetilde{B})$ (которые могут быть заданы на различных фильтрованных вероятностных пространствах) выполнено $\operatorname{Law}\left(X_{t} ; t \geqslant 0\right)=\operatorname{Law}\left(\widetilde{X}_{t} ; t \geqslant 0\right)$.

О п р е д е л е н и е 1.4. Для уравнения (1.1) имеет место сильная единственность, если для любых решений $(X, B)$ и $(\widetilde{X}, B)$ (заданных на одном и том же фильтрованном вероятностном пространстве) выполнено $\mathbf{P}\left\{\forall t \geqslant 0, X_{t}=\tilde{X}_{t}\right\}=1$. 
3 а м е ч а н и я. (i) Если не существует решения уравнения (1.1), то имеют место и сильная, и слабая единственность.

(ii) Обзоры достаточных условий для различных видов существования решения и различных видов единственности можно найти в $[7$, гл. 4 , $\S 4],[8$, гл. IX], [10], [12].

Следуюшие два предложения проясняют преимущества сильного существования и сильной единственности.

Предложение 1.1. Пусть $(X, B)$ - сильное решение уравнения (1.1). Тогда:

(i) существует измеримое отображение

$$
\Phi:\left(C\left(\mathbf{R}_{+}, \mathbf{R}^{m}\right), \mathscr{B}\right) \longrightarrow\left(C\left(\mathbf{R}_{+}, \mathbf{R}^{n}\right), \mathscr{B}\right)
$$

$(\mathscr{B}$ обозначает борелевскую $\sigma$-алгебру) такое, что $X(\omega)=\Phi(B(\omega))$ для P-n.6. $\omega$;

(ii) если $\widetilde{B}-(\widetilde{\mathscr{F}}, \widetilde{\mathbf{P}})-\mathrm{BM}^{m}(0)$ u $\widetilde{X}(\widetilde{\omega}):=\Phi(\widetilde{B}(\widetilde{\omega}))$, mo $(\widetilde{X}, \widetilde{B})$ sвляется сильным решением уравнения $(1.1)$ на $\left(\widetilde{\Omega}, \widetilde{F},\left(\widetilde{F}_{t}\right), \widetilde{\mathbf{P}}\right)$.

Доказательство содержится, например, в [1].

Предложение 1.2 (Ямада, Ватанабэ). Предположим, что для (1.1) имеет место сильная единственность. Тогда

(i) для (1.1) имеет место слабая единственность;

(ii) существует измеримое отображение

$$
\Phi:\left(C\left(\mathbf{R}_{+}, \mathbf{R}^{m}\right), \mathscr{B}\right) \longrightarrow\left(C\left(\mathbf{R}_{+}, \mathbf{R}^{n}\right), \mathscr{B}\right)
$$

такое, что для любого решения $(X, B)$ уравнения (1.1) выполнено $X(\omega)=\Phi(B(\omega)) \partial \Omega$ P $\mathbf{P}$-n.в. $\omega$.

Доказательство содержится в [11] (см. также [8, гл. IX, (1.7)]).

Ситуацию с решениями СДУ можно описать следующим образом.

Существуют СДУ вида (1.1), для которых нет решения ни на одном фильтрованном вероятностном пространстве (см. пример 2.1). Существуют СДУ вида (1.1), для которых на некоторых фильтрованных вероятностных пространствах есть решение (или даже несколько решений с одним и тем же броуновским движением), в то время как на других фильтрованных вероятностных пространствах нет решений (см. пример 2.2).

Если хотя бы на одном фильтрованном вероятностном пространстве нашлось сильное решение (1.1), то и на любом другом фильтрованном вероятностном пространстве с любым броуновским движением на нем существует сильное решение (см. предложение 1.1). При этом, однако, может существовать несколько решений (см. пример 2.3).

Если же для (1.1) имеет место сильная единственность и существует решение на некотором фильтрованном вероятностном пространстве, 
то на любом фильтрованном вероятностном пространстве с любым броуновским движением на нем сушествует в точности одно решение, и оно является сильным (см. предложение 1.2). Это наилучшая возможная ситуация.

Итак, согласно теореме Ямада-Ватанабэ (предложение 1.2), сильная единственность и слабое существование гарантируют, что ситуация является наилучшей.

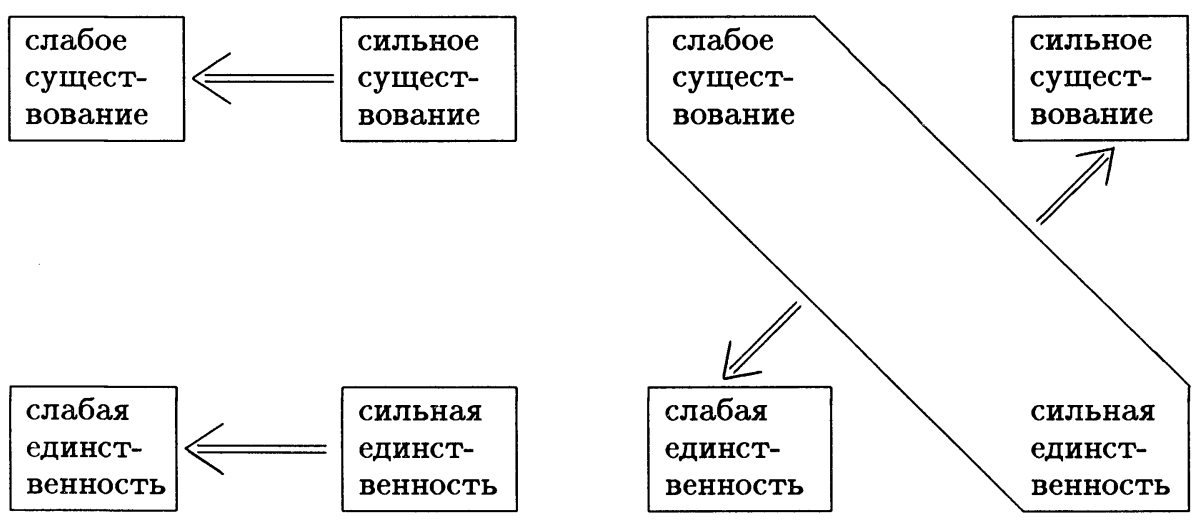

Диаграмма 1. Очевидные импликации и импликации, вытекающие из теоремы Ямада-Ватанабэ

В настоящей статье показано, что слабая единственность и сильное существование также гарантируют наилучшую ситуацию. Именно, мы доказываем, что из этих двух свойств вытекает сильная единственность (теорема 3.2). Доказательство основывается на результате, представляющем самостоятельный интерес: если для (1.1) имеет место слабая единственность, то совместное распределение $\operatorname{Law}\left(X_{t}, B_{t} ; t \geqslant 0\right)$ является одним и тем же для всех решений $(X, B)$ (теорема 3.1).

3 а м е ч а н и я. (i) Существуют исследования уравнений более общего вида, чем (1.1). Это СДУ, в которых вместо броуновского движения $B$ стоит (заданный) семимартингал $Z$. Для таких уравнений слабая единственность иногда определяется как единственность совместного распределения пары $(X, Z)$ (см. [4], [5]). Теорема 3.1 показывает, что для СДУ вида (1.1) эта усиленная форма слабой единственности эквивалентна определению 1.3 .

(ii) В статье [2] Х.-Ю. Энгельберт доказал, что единственность совместного распределения $\operatorname{Law}\left(X_{t}, B_{t} ; t \geqslant 0\right)$ и сильное существование гарантируют сильную единственность. Кроме того, в [2] доказано при некоторых дополнительных предположениях, что из слабой единственности вытекает единственность совместного распределения $(X, B)$. Тео- 
рема 3.1 настоящей работы показывает, что это утверждение верно без всяких дополнительных предположений.

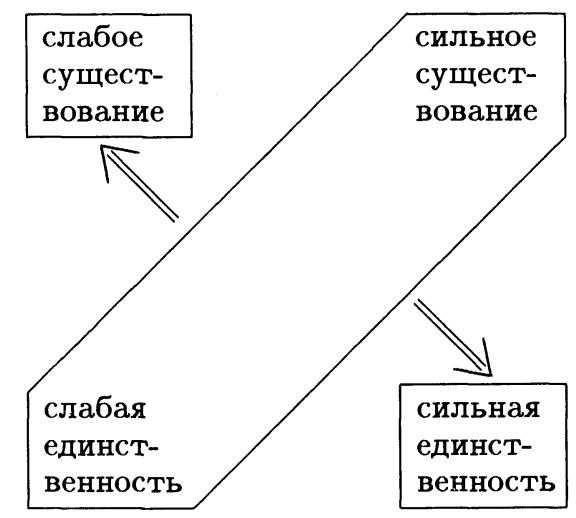

Диаграмма 2. Импликации, вытекающие из теоремы 3.2

Работа построена следующим образом. В п. 2 приводятся некоторые примеры СДУ. Эти примеры иллюстрируют различные возможные ситуации с существованием и единственностью решений. Примеры 2.2 и 2.3 хорошо известны. Основные результаты работы содержатся в п. 3 . Пункт 4 содержит интерпретацию теоремы 3.1 в терминах мартингальных проблем. Кроме того, в п. 4 приводится таблица, показывающая, какие комбинации существования и единственности возможны, а какие невозможны (см. таблицу 1).

\section{2. Примеры.}

Пример 2.1 (нет решения). Для стохастического дифференциального уравнения

$$
d X_{t}=-\frac{1}{2 X_{t}} I\left(X_{t} \neq 0\right) d t+d B_{t}, \quad X_{0}=0
$$

не существует решения.

Д ок аз ат ельст в о. Предположим, что $(X, B)$ - решение (2.1). Тогда

$$
X_{t}=-\int_{0}^{t} \frac{1}{2 X_{s}} I\left(X_{s} \neq 0\right) d s+B_{t}, \quad t \geqslant 0 .
$$

По формуле Ито,

$$
\begin{aligned}
X_{t}^{2} & =-\int_{0}^{t} 2 X_{s} \frac{1}{2 X_{s}} I\left(X_{s} \neq 0\right) d s+\int_{0}^{t} 2 X_{s} d B_{s}+\int_{0}^{t} 1 d s \\
& =\int_{0}^{t} I\left(X_{s}=0\right) d s+\int_{0}^{t} 2 X_{s} d B_{s}, \quad t \geqslant 0 .
\end{aligned}
$$


Процесс $X$ является непрерывным семимартингалом с $\langle X\rangle_{t}=t$. Поэтому (см. $[8$, гл. VI, (1.6)])

$$
\int_{0}^{t} I\left(X_{s}=0\right) d s=\int_{\mathbf{R}} I(x=0) L_{t}^{x}(X) d x=0, \quad t \geqslant 0
$$

где $L_{t}^{x}(X)$ обозначает локальное время, проведенное процессом $X$ в точке $x$ к моменту времени $t$. Следовательно, $X^{2}$ - локальный мартингал. Поскольку $X^{2} \geqslant 0$ и $X_{0}^{2}=0$, заключаем, что $X^{2}=0$ п.н. Но это означает, что $(X, B)$ не является решением уравнения (2.1).

Пример 2.2 (нет сильного решения и сильной единственности; Танака). Для стохастического дифференциального уравнения

$$
d X_{t}=\operatorname{sign} X_{t} d B_{t}, \quad X_{0}=0,
$$

zдe

$$
\text { sign } x=\left\{\begin{array}{cl}
1, & \text { если } x>0, \\
-1, & \text { если } x \leqslant 0,
\end{array}\right.
$$

существует решение $и$ имеет место слабая единственность, но не существует сильного решения и нет сильной единственности.

Д ок а а т ельст в о. Пусть $W$ - броуновское движение на некотором пространстве $(\Omega, \mathscr{F}, \mathbf{P})$. Положим $X_{t}=W_{t}, B_{t}=$ $\int_{0}^{t} \operatorname{sign} W_{s} d W_{s}, t \geqslant 0$, и возьмем $\mathscr{F}_{t}=\mathscr{F}_{t}^{B}$. Тогда $(X, B)$ является решением уравнения $(2.2)$ на $\left(\Omega, \mathscr{F},\left(\mathscr{F}_{t}\right), \mathbf{P}\right)$.

Если $(X, B)$ - решение (2.2) на некотором фильтрованном вероятностном пространстве $\left(\Omega, \mathscr{F},\left(\mathscr{F}_{t}\right), \mathbf{P}\right)$, то $X$ является непрерывным $\left(\mathscr{F}_{t}, \mathbf{P}\right)$-локальным мартингалом с $\langle X\rangle_{t}=t$. Из теоремы Леви (см. [8, гл. IV, (3.6)]) вытекает, что $X$ является броуновским движением. Это означает слабую единственность.

Если $(X, B)$ - решение $(2.2)$, то $B_{t}=\int_{0}^{t} \operatorname{sign} X_{s} d X_{s}, t \geqslant 0$. Отсюда вытекает, что $\mathscr{F}_{t}^{B}=\mathscr{F}_{t}^{|X|}$ (см. [8, гл. VI, (2.2)]). Следовательно, не сушествует сильного решения.

Если $(X, B)$ - решение $(2.2)$, то $(-X, B)$ также является решением. Итак, сильная единственность не имеет места.

3 а м е ч а н и е. Пусть $B-$ броуновское движение на $(\Omega, \mathscr{F}, \mathbf{P})$. Положим $\mathscr{F}_{t}=\mathscr{F}_{t}^{B}$. Тогда на $\left(\Omega, \mathscr{F},\left(\mathscr{F}_{t}\right), \mathbf{P}\right)$ не существует решения уравнения (2.2) с броуновским движением $B$. Действительно, если $(X, B)$ является решением, то процесс $X\left(\mathscr{F}_{t}\right)$-согласован и, следовательно, $(X, B)$ является сильным решением. С другой стороны, уравнение (2.2) не имеет сильных решений.

Пример 2.3 (нет единственности). Для стохастического дифференциального уравнения

$$
d X_{t}=I\left(X_{t} \neq 0\right) d B_{t}, \quad X_{0}=0
$$

существует сильное решение, но нет ни слабой, ни сильной единственности. 
Д ок аз а т е льст во. Достаточно заметить, что $(B, B)$ и $(0, B)$ - решения уравнения $(2.3)$, если $B$ - броуновское движение на фильтрованном вероятностном пространстве $\left(\Omega, \mathscr{F},\left(\mathscr{F}_{t}\right), \mathbf{P}\right)$.

З а м е ч а и е. Пусть $B-$ броуновское движение, а $\xi$ - случайная величина, независимая от $B$ и имеющая распределение $\mathbf{P}\{\xi=1\}=$ $\mathbf{P}\{\xi=-1\}=\frac{1}{2}$. Положим

$$
X_{t}(\omega)= \begin{cases}B_{t}(\omega), & \text { если } \xi(\omega)=1, \\ 0, & \text { если } \xi(\omega)=-1,\end{cases}
$$

и возьмем $\mathscr{F}_{t}=\mathscr{F}_{t}^{X}$. Тогда $(X, B)$ - решение уравнения $(2.3)$, не являющееся сильным. Действительно, для любого $t>0$ случайная величина $\xi$ является $\sigma\left(X_{t}\right)$-измеримой, в то время как $\xi$ не является $\mathscr{F}_{t}^{B}$-измеримой.

Пример 2.4 (нет сильного существования и нет единственности). Для стохастического дифференциального уравнения

$$
d X_{t}=I\left(X_{t} \neq 1\right) \operatorname{sign} X_{t} d B_{t}, \quad X_{0}=0
$$

существует решение, но не существует сильного решения и нет ни слабой, ни сильной единственности.

Доказательст в о. Если $W$ - броуновское движение, то пара

$$
X_{t}=W_{t}, \quad B_{t}=\int_{0}^{t} \operatorname{sign} W_{s} d W_{s}
$$

является решением уравнения (2.4).

Пусть $(X, B)$ - решение, определенное по формуле (2.5). Положим $\tau=\inf \left\{t \geqslant 0: X_{t}=1\right\}, \tilde{X}_{t}=X_{t \wedge \tau}$. Тогда $(\tilde{X}, B)-$ другое решение. Итак, нет ни слабой, ни сильной единственности.

Предположим, что $(X, B)$ - сильное решение уравнения (2.4). Положим $\tau=\inf \left\{t \geqslant 0: X_{t}=1\right\}, X_{t}^{\tau}=X_{t \wedge \tau}, B_{t}^{\tau}=B_{t \wedge \tau}$. Случайная величина $\tau^{\prime}=\inf \left\{t \geqslant 0: X_{t}=\frac{1}{2}\right\}$ является $\left(\mathscr{F}_{t}^{X}\right)$-моментом остановки. Поскольку процесс $X\left(\overline{\mathscr{F}}_{t}^{B}\right)$-согласован, то $\tau^{\prime}$ также является $\left(\overline{\mathscr{F}}_{t}^{B}\right)$-моментом остановки. Тогда существует $\left(\mathscr{F}_{t}^{B}\right)$-момент остановки $\tau^{\prime \prime}$ такой, что $\tau^{\prime \prime}=\tau^{\prime}$ п.н. (см. [6, гл. I, (1.19)]). Согласно критерию Гальмарино (см. [3, $\S 3.2]), \tau^{\prime \prime}$ также является $\left(\mathscr{F}_{t}^{B^{\tau}}\right)$-моментом остановки. С другой стороны, $B_{t}^{\tau}=\int_{0}^{t} \operatorname{sign} X_{t}^{\tau} d X_{t}^{\tau}, \quad t \geqslant 0$. Согласно теории локальных времен для непрерывных семимартингалов, из этого равенства вытекает, что $\mathscr{F}_{t}^{B^{\tau}} \subseteq \mathscr{F}_{t}^{\left|X^{\tau}\right|}$ (см. [8, гл. VI, § 1]). Но, очевидно, $\tau^{\prime}, \tau^{\prime \prime}$ не являются моментами остановки относительно фильтрации $\left(\mathscr{F}_{t}^{\left|X^{\tau}\right|}\right)$. Итак, у (2.4) не существует сильного решения.

\section{3. Основные результаты.}

Теорема 3.1. Предположим, что для (1.1) имеет место слабая единственность. Тогда для любых решений $(X, B)$ и $(\widetilde{X}, \widetilde{B})$ (которые могут быть заданы на различных фильтрованных вероятностных пространствах) выполнено $\operatorname{Law}\left(X_{t}, B_{t} ; t \geqslant 0\right)=\operatorname{Law}\left(\widetilde{X}_{t}, \widetilde{B}_{t} ; t \geqslant 0\right)$. 
Теорема 3.2. Предположим, что для (1.1) имеют место слабая единственность и сильное существование. Тогда для (1.1) имеет место сильная единственность.

З а м е ч а н и е. Предположим, что для СДУ (1.1) $n=m=1$, а $\sigma_{t}(x) \neq 0$ для любых $t \geqslant 0, x \in C\left(\mathbf{R}_{+}, \mathbf{R}\right)$. В этом случае теорема 3.1 практически тривиальна. Достаточно заметить, что $B$ является измеримым функционалом $X$ :

$$
B_{t}=\int_{0}^{t} \frac{1}{\sigma_{s}(X)} d M_{s}
$$

где $M_{t}=X_{t}-\int_{0}^{t} b_{s}(X) d s$.

Если же $\sigma$ может принимать нулевое значение, то такое рассуждение не проходит.

Для доказательства теорем 3.1 и 3.2 нам потребуется несколько вспомогательных лемм.

Лемма 3.1. Если $B-\left(\mathscr{F}_{t}, \mathbf{P}\right)-\mathrm{BM}^{m}(0)$, то для любых $0 \leqslant s \leqslant t$ случайная величина $B_{t}-B_{s}$ независима от $\mathscr{F}_{s}$.

Д ок аз а т е ль с т о. Согласно формуле Ито, для любого $\lambda \in \mathbf{R}^{m}$ процесс $\exp \left\{i\left(\lambda, B_{t}\right)+\|\lambda\|^{2} t / 2\right\}$ является $\left(\mathscr{F}_{t}, \mathbf{P}\right)$-локальным мартингалом. Поскольку он ограничен, то является мартингалом. Поэтому для любых $0 \leqslant s \leqslant t, A \in \mathscr{F}_{s}, \lambda \in \mathbf{R}^{m}$ выполнено

$$
\mathbf{E}\left[\exp \left\{i\left(\lambda, B_{t}-B_{s}\right)\right\} I_{A}\right]=\exp \left\{-(t-s) \frac{\|\lambda\|^{2}}{2}\right\} \mathbf{P}(A) .
$$

Отсюда вытекает требуемый результат.

Лемма 3.2. Пусть $t \geqslant 0 u f \in L^{2}([0, t])$. Для $k \in \mathbf{N}$ положим

$$
f^{(k)}(s)= \begin{cases}0, & \text { если } s \in\left[0, \frac{t}{k}\right], \\ \frac{k}{t} \int_{(i-1) t / k}^{i t / k} f(r) d r, & \text { если } s \in\left(\frac{i t}{k}, \frac{(i+1) t}{k}\right], i=1, \ldots, k-1 .\end{cases}
$$

Тогда $f^{(k)} \rightarrow f$ в $L^{2}([0, t]) n p u k \rightarrow \infty$.

Д ок а з а те л ь с т в о. Имеем

$$
\begin{aligned}
\left\|f^{(k)}\right\|_{L^{2}([0, t])}^{2} & =\sum_{i=1}^{k-1} \frac{t}{k}\left(\frac{k}{t} \int_{(i-1) t / k}^{i t / k} f(r) d r\right)^{2} \\
& \leqslant \sum_{i=1}^{k-1} \int_{(i-1) t / k}^{i t / k} f^{2}(r) d r \leqslant \int_{0}^{t} f^{2}(r) d r=\|f\|_{L^{2}([0, t])}^{2} .
\end{aligned}
$$

Фиксируем $\varepsilon>0$. Можно найти функцию $\varphi \in C([0, t])$ такую, что $\|\varphi-f\|_{L^{2}([0, t])}<\varepsilon$. Обозначим через $\varphi^{(k)}$ функцию, построенную по $\varphi$ так же, как $f^{(k)}$ построена по $f$. С учетом (3.1) имеем $\left\|\varphi^{(k)}-f^{(k)}\right\|_{L^{2}([0, t])} \leqslant\|\varphi-f\|_{L^{2}([0, t])}<\varepsilon$ для любого $k \in \mathbf{N}$. Поскольку $\varphi$ 
непрерывна, существует $K \in \mathbf{N}$ такое, что для любого $k \geqslant K$ выполнено $\left\|\varphi^{(k)}-\varphi\right\|_{L^{2}([0, t])}<\varepsilon$. Отсюда вытекает требуемый результат.

Напомним теперь следующий общий факт из теории меры. Пусть $\xi: \Omega \rightarrow E-$ случайный элемент на $(\Omega, \mathscr{F}, \mathbf{P})$, принимающий значения в польском пространстве $(E, \mathscr{B}(E))$. Пусть $\mathscr{G} \subseteq \mathscr{F}$. Тогда существует условное распределение $\xi$ относительно $\mathscr{G}$, т.е. семейство $\left(\mathbf{Q}_{\omega}\right)_{\omega \in \Omega}$ вероятностных мер на $(E, \mathscr{B}(E))$ такое, что

i) для любого $A \in \mathscr{B}(E)$ отображение $\omega \mapsto \mathbf{Q}_{\omega}(A) \mathscr{G}$-измеримо;

ii) для любых $A \in \mathscr{B}(E), D \in \mathscr{G}$

$$
\mathbf{P}(D \cap\{\xi \in A\})=\int_{D} \mathbf{Q}_{\omega}(A) \mathbf{P}(d \omega) .
$$

Условное распределение единственно в следующем смысле: если $\left(\widetilde{\mathbf{Q}}_{\omega}\right)_{\omega \in \Omega}-$ другое семейство с теми же свойствами, то $\mathbf{Q}_{\omega}=\widetilde{\mathbf{Q}}_{\omega}$ для P-п.в. $\omega$.

З а м е ч а н и е. Свойства i), ii) означают, что для любой $\mathscr{B}(E)$ измеримой ограниченной функции $h$ случайная величина $\eta(\omega):=\mathbf{E}_{\mathbf{Q}_{\omega}}[h]$ является версией $\mathbf{E}_{\mathbf{P}}[h(\xi) \mid \mathscr{G}]$. Заметим также, что если для множества $A \in \mathscr{B}(E)$ выполнено свойство $\mathbf{P}\{\xi \in A\}=1$, то $\mathbf{Q}_{\omega}(A)=1$ для $\mathbf{P}$-п.в. $\omega$.

Лемма 3.3. Пусть $(X, B)$ - решение (1.1) на фильтрованном вероятностном пространстве $\left(\Omega, \mathscr{F},\left(\mathscr{F}_{t}\right), \mathbf{P}\right)$. Пусть $\left(\mathbf{Q}_{\omega}\right)_{\omega \in \Omega}-$ условное распределение $(X, B)$ относительно $\mathscr{F}_{0}$ (здесь $(X, B)$ рассматривается как $C\left(\mathbf{R}_{+}, \mathbf{R}^{n+m}\right)$-значный случайный элемент). Обозначим через $Y$ прочесс, состоящий из первых $n$ координат канонического прочесса на $C\left(\mathbf{R}_{+}, \mathbf{R}^{n+m}\right)$, и через $Z$ прочесс, состоящий из последних $m$ координат. Пусть $\left(\mathscr{H}_{t}\right)-$ каноническая фильтрачия на $C\left(\mathbf{R}_{+}, \mathbf{R}^{n+m}\right)$ u $\mathscr{H}=\bigvee_{t \geqslant 0} \mathscr{H}_{t}$. Тогда для $\mathbf{P}$-n.в. $\omega$ пара $(Y, Z)$ является решением (1.1) на $\left(C\left(\mathbf{R}_{+}, \mathbf{R}^{n+m}\right), \mathscr{H},\left(\mathscr{H}_{t}\right), \mathbf{Q}_{\omega}\right)$.

Д ок а з а т е л с т в о. Проверим выполнение условий i)-iii) определения 1.1 .

i) Для любых $0 \leqslant s \leqslant t, D \in \mathscr{H}_{s}, \lambda \in \mathbf{R}^{m}, A \in \mathscr{F}_{0}$ выполнено

$$
\begin{aligned}
& \mathbf{E}_{\mathbf{P}}\left[\exp \left\{i\left(\lambda, B_{t}-B_{s}\right)\right\} I((X, B) \in D) I_{A}\right] \\
& \quad=\exp \left\{-(t-s) \frac{\|\lambda\|^{2}}{2}\right\} \mathbf{E}_{\mathbf{P}}\left[I((X, B) \in D) I_{A}\right] .
\end{aligned}
$$

Следовательно, для любых $0 \leqslant s \leqslant t, D \in \mathscr{H}_{s}, \lambda \in \mathbf{R}^{m}$ имеем

$$
\mathbf{E}_{\mathbf{Q}_{\omega}}\left[\exp \left\{i\left(\lambda, Z_{t}-Z_{s}\right)\right\} I_{D}\right]=\exp \left\{-(t-s) \frac{\|\lambda\|^{2}}{2}\right\} \mathbf{Q}_{\omega}\left(I_{D}\right)
$$

для $\mathbf{P}$-п.в. $\omega$. Выбирая счетный набор $\left\{s_{k}, t_{k}, D_{k l}, \lambda_{k l} ; k, l \in \mathbf{N}\right\}$ таким образом, что последовательность $\left(s_{k}, t_{k}\right)$ пробегает все пары неотрицательных рациональных чисел $\left(s_{k} \leqslant t_{k}\right)$, совокупность $\left\{D_{k l} ; l \in \mathbf{N}\right\}$ порождает $\mathscr{H}_{s_{k}}$, а множество $\left\{\lambda_{k l} ; l \in \mathbf{N}\right\}$ плотно в $\mathbf{R}^{m}$, заключаем, что для $\mathbf{P}$-п.в. $\omega$ процесс $Z$ является $\left(\mathscr{H}_{t}, \mathbf{Q}_{\omega}\right)$--BM ${ }^{m}(0)$. 
ii) Для любого $t \geqslant 0$

$$
\int_{0}^{t}\left(\sum_{i=1}^{n}\left|b_{s}^{i}(X)\right|+\sum_{i=1}^{n} \sum_{j=1}^{m}\left(\sigma_{s}^{i j}(X)\right)^{2}\right) d s<\infty \quad \text { Р-п.н. }
$$

Следовательно, для любого $t \geqslant 0$

$$
\int_{0}^{t}\left(\sum_{i=1}^{n}\left|b_{s}^{i}(Y)\right|+\sum_{i=1}^{n} \sum_{j=1}^{m}\left(\sigma_{s}^{i j}(Y)\right)^{2}\right) d s<\infty \quad \mathbf{Q}_{\omega} \text {-п.н. }
$$

для Р-п.в. $\omega$.

iii) Фиксируем $t \geqslant 0$. Для $k \in \mathbf{N}$ определим процесс

$$
\sigma^{(k)}: \mathbf{R}_{+} \times C\left(\mathbf{R}_{+}, \mathbf{R}^{n}\right) \ni(s, y) \longmapsto \sigma_{s}^{(k)}(y) \in \mathbf{R}
$$

по формуле

$$
\sigma_{s}^{(k)}(y)=\left\{\begin{array}{lll}
0, & \text { если } & s \in\left[0, \frac{t}{k}\right], \\
\frac{k}{t} \int_{(i-1) t / k}^{i t / k} \sigma_{r}(y) d r, & \text { если } & s \in\left(\frac{i t}{k}, \frac{(i+1) t}{k}\right], \\
& i=1, \ldots, k-1 .
\end{array}\right.
$$

Согласно лемме 3.2 ,

$$
\int_{0}^{t}\left\|\sigma_{s}^{(k)}(X)-\sigma_{s}(X)\right\|^{2} d s \underset{k \rightarrow \infty}{\stackrel{\mathbf{P}-\text { п.н. }}{\longrightarrow}} 0 .
$$

Следовательно,

$$
\int_{0}^{t} \sigma_{s}^{(k)}(X) d B_{s} \underset{k \rightarrow \infty}{\stackrel{\mathbf{P}}{\longrightarrow}} \int_{0}^{t} \sigma_{s}(X) d B_{s}
$$

что означает сходимость

$$
\sum_{i=2}^{k} \sigma_{i t / k}^{(k)}(X)\left(B_{i t / k}-B_{(i-1) t / k}\right) \underset{k \rightarrow \infty}{\stackrel{\mathbf{P}}{\longrightarrow}} X_{t}-x-\int_{0}^{t} b_{s}(X) d s
$$

(здесь использована векторная форма записи). Существует подпоследовательность $(k(l))$ такая, что вдоль нее сходимость в $(3.3)$ имеет место Р-п.н. Поэтому

$$
\sum_{i=2}^{k(l)} \sigma_{i t / k(l)}^{(k(l))}(Y)\left(Z_{i t / k(l)}-Z_{(i-1) t / k(l)}\right) \underset{l \rightarrow \infty}{\stackrel{\mathbf{Q}_{\omega} \text {-п.н. }}{\longrightarrow}} Y_{t}-x-\int_{0}^{t} b_{s}(Y) d s
$$

для Р-п.в. $\omega$. С другой стороны, в силу (3.2)

$$
\int_{0}^{t}\left\|\sigma_{s}^{(k)}(Y)-\sigma_{s}(Y)\right\|^{2} d s \underset{k \rightarrow \infty}{\stackrel{\mathbf{Q}_{\omega}-\text { п.н. }}{\longrightarrow}} 0
$$

для Р-п.в. $\omega$, откуда

$$
\int_{0}^{t} \sigma_{s}^{(k)}(Y) d Z_{s} \underset{k \rightarrow \infty}{\stackrel{\mathbf{Q}_{\omega}}{\longrightarrow}} \int_{0}^{t} \sigma_{s}(Y) d Z_{s}
$$


для Р-п.в. $\omega$. С учетом (3.4) и (3.5), получаем

$$
Y_{t}-x-\int_{0}^{t} b_{s}(Y) d s=\int_{0}^{t} \sigma_{s}(Y) d Z_{s} \quad \mathbf{Q}_{\omega} \text {-П.н. }
$$

для Р-п.в. $\omega$. Это завершает доказательство.

Доказ а тельст во те о ремы 3.1. Пусть $(X, B)$ - решение уравнения (1.1) на фильтрованном вероятностном пространстве $\left(\Omega, \mathscr{F},\left(\mathscr{F}_{t}\right), \mathbf{P}\right)$. Пусть $\left(W_{t}\right)_{t \geqslant 0}$ и $\left(\bar{W}_{t}\right)_{t \geqslant 0}-$ независимые $\left(\mathscr{F}_{t}^{\prime}, \mathbf{P}^{\prime}\right)-\mathrm{BM}^{m}(0)$. Положим

$$
\left(\widetilde{\Omega}, \widetilde{\mathscr{F}},\left(\widetilde{\mathscr{F}}_{t}\right), \widetilde{\mathbf{P}}\right)=\left(\Omega, \mathscr{F},\left(\mathscr{F}_{t}\right), \mathbf{P}\right) \times\left(\Omega^{\prime}, \mathscr{F}^{\prime},\left(\mathscr{F}_{t}^{\prime}\right), \mathbf{P}^{\prime}\right)
$$

Процессы $X, B, W, \bar{W}$ определяются на $\widetilde{\Omega}$ очевидным образом. Пара $(X, B)$ является решением уравнения $(1.1)$ на $\left(\widetilde{\Omega}, \widetilde{\mathscr{F}},\left(\widetilde{\mathscr{F}}_{t}\right), \widetilde{\mathbf{P}}\right)$, а $W, \bar{W}$ являются независимыми $\left(\widetilde{\mathscr{F}}_{t}, \widetilde{\mathbf{P}}\right)-\mathrm{BM}^{m}(0)$.

Для любых $t \geqslant 0, y \in C\left(\mathbf{R}_{+}, \mathbf{R}^{n}\right)$ матрица $\sigma_{t}(y)$ соответствует линейному оператору $\mathbf{R}^{m} \rightarrow \mathbf{R}^{n}$. Обозначим через $\varphi_{t}(y) m \times m$-матрицу оператора ортогональной проекции на $\left(\operatorname{ker} \sigma_{t}(y)\right)^{\perp} ;$ обозначим через $\psi_{t}(y)$ $m \times m$-матрицу оператора ортогональной проекции на $\operatorname{ker} \sigma_{t}(y)$. Процессы $\varphi=\varphi_{t}(y)$ и $\psi=\psi_{t}(y)$ являются предсказуемыми $\mathbf{R}^{m \times m}$-значными процессами на $C\left(\mathbf{R}_{+}, \mathbf{R}^{n}\right)$.

\section{Положим}

$$
\begin{aligned}
& U_{t}=\int_{0}^{t} \varphi_{s}(X) d B_{s}+\int_{0}^{t} \psi_{s}(X) d W_{s} \\
& V_{t}=\int_{0}^{t} \varphi_{s}(X) d \bar{W}_{s}+\int_{0}^{t} \psi_{s}(X) d B_{s}
\end{aligned}
$$

Тогда $2 m$-мерный процесс $(U, V)$ является непрерывным $\left(\widetilde{\mathscr{F}}_{t}, \widetilde{\mathbf{P}}\right)$-локальным мартингалом. Для любых $i, j=1, \ldots, m$, с учетом симметричности матриц $\varphi_{t}(y), \psi_{t}(y)$, имеем

$$
\begin{aligned}
\left\langle U^{i}, U^{j}\right\rangle_{t} & =\int_{0}^{t}\left(\sum_{k=1}^{m} \varphi_{s}^{i k}(X) \varphi_{s}^{j k}(X)+\sum_{k=1}^{m} \psi_{s}^{i k}(X) \psi_{s}^{j k}(X)\right) d s \\
& =\int_{0}^{t}\left(\left(\varphi_{s}(X) e_{i}, \varphi_{s}(X) e_{j}\right)+\left(\psi_{s}(X) e_{i}, \psi_{s}(X) e_{j}\right)\right) d s \\
& =\int_{0}^{t}\left(\varphi_{s}(X) e_{i}+\psi_{s}(X) e_{i}, \varphi_{s}(X) e_{j}+\psi_{s}(X) e_{j}\right) d s \\
& =\int_{0}^{t}\left(e_{i}, e_{j}\right) d s=\delta_{i j} t
\end{aligned}
$$

где $\left(e_{i}\right)_{i=1}^{m}-$ стандартный базис в $\mathbf{R}^{m}$. Аналогично,

$$
\begin{aligned}
\left\langle U^{i}, V^{j}\right\rangle_{t} & =\int_{0}^{t}\left(\varphi_{s}(X) e_{i}, \psi_{s}(X) e_{j}\right) d s=0, \\
\left\langle V^{i}, V^{j}\right\rangle_{t} & =\delta_{i j} t .
\end{aligned}
$$


Согласно теореме Леви (см. [8, гл. IV, (3.6)]), заключаем, что процесс $(U, V)$ является $\left(\widetilde{\mathscr{F}}_{t}, \widetilde{\mathbf{P}}\right)-\mathrm{BM}^{2 m}(0)$.

Для любого $t \geqslant 0$ выполнено

$$
\begin{aligned}
\int_{0}^{t} \sigma_{s}(X) d B_{s} & =\int_{0}^{t}\left(\sigma_{s}(X) \varphi_{s}(X)\right) d B_{s}=\int_{0}^{t} \sigma_{s}(X) d\left(\int_{0}^{s} \varphi_{r}(X) d B_{r}\right) \\
& =\int_{0}^{t} \sigma_{s}(X) d\left(\int_{0}^{s} \varphi_{r}(X) d U_{r}\right)=\int_{0}^{t} \sigma_{s}(X) d U_{s}
\end{aligned}
$$

где $\sigma_{s}(X) \varphi_{s}(X)$ обозначает произведение матриц. Следовательно, $(X, U)$ - решение уравнения $(1.1)$ на $\left(\widetilde{\Omega}, \widetilde{\mathscr{F}},\left(\widetilde{\mathscr{F}}_{t}\right), \widetilde{\mathbf{P}}\right)$.

Рассмотрим фильтрацию

$$
\mathscr{G}_{s}=\widetilde{\mathscr{F}}_{s} \vee \sigma\left(V_{t} ; t \geqslant 0\right)=\widetilde{\mathscr{F}}_{s} \vee \sigma\left(V_{t}-V_{s} ; t \geqslant s\right), \quad s \geqslant 0 .
$$

Из леммы 3.1 вытекает, что для любого $s \geqslant 0 \sigma$-алгебры $\widetilde{\mathscr{F}}_{s}$ и $\sigma\left(U_{t}-\right.$ $\left.U_{s} ; t \geqslant s\right) \vee \sigma\left(V_{t}-V_{s} ; t \geqslant s\right)$ независимы. Поэтому для любых $0 \leqslant s \leqslant t$, $i=1, \ldots, m, A \in \widetilde{\mathscr{F}}_{s}, D \in \sigma\left(V_{t}-V_{s} ; t \geqslant s\right)$ имеем

$\mathbf{E}_{\widetilde{\mathbf{P}}}\left[\left(U_{t}^{i}-U_{s}^{i}\right) I_{D} I_{A}\right]=\mathbf{E}_{\widetilde{\mathbf{P}}}\left[\left(U_{t}^{i}-U_{s}^{i}\right) I_{D}\right] \tilde{\mathbf{P}}(A)=\mathbf{E}_{\widetilde{\mathbf{P}}}\left[U_{t}^{i}-U_{s}^{i}\right] \widetilde{\mathbf{P}}(D) \widetilde{\mathbf{P}}(A)=0$.

Следовательно, $U$ является $\left(\mathscr{G}_{t}, \tilde{\mathbf{P}}\right)-\mathrm{BM}^{m}(0)$. Поскольку стохастический интеграл $\int_{0}^{t} \sigma_{s}(X) d U_{s}$ одинаков относительно фильтраций $\left(\widetilde{\mathscr{F}}_{t}\right)$ и $\left(\mathscr{G}_{t}\right)$, пара $(X, U)$ является решением уравнения $(1.1)$ на $\left(\widetilde{\Omega}, \widetilde{\mathscr{F}},\left(\mathscr{G}_{t}\right), \widetilde{\mathbf{P}}\right)$.

Пусть $\left(\mathbf{Q}_{\tilde{\omega}}\right)_{\tilde{\omega} \in \tilde{\Omega}}-$ условное распределение $(X, U)$ относительно $\mathscr{G}_{0}$. По лемме 3.3 , пара $(Y, Z)$ является решением уравнения (1.1) на $\left(C\left(\mathbf{R}_{+}, \mathbf{R}^{n+m}\right), \mathscr{H},\left(\mathscr{H}_{t}\right), \mathbf{Q}_{\tilde{\omega}}\right)$ для $\widetilde{\mathbf{P}}$-п.в. $\widetilde{\omega}$. Поскольку для $(1.1)$ имеет место слабая единственность, заключаем, что распределение $\operatorname{Law}\left(Y_{t} ; t \geqslant 0 \mid \mathbf{Q}_{\tilde{\omega}}\right)$ (которое является условным распределением $X$ относительно $\mathscr{G}_{0}$ ) одно и то же для $\widetilde{\mathbf{P}}$-п.в. $\widetilde{\omega}$. Это означает независимость процесса $X$ от $\mathscr{G}_{0}$. В частности, $X$ и $V$ независимы.

Для любых $t \geqslant 0, y \in C\left(\mathbf{R}_{+}, \mathbf{R}^{n}\right)$ сужение оператора $\sigma_{t}(y)$ на $\left(\operatorname{ker} \sigma_{t}(y)\right)^{\perp}$ является биекцией из $\left(\operatorname{ker} \sigma_{t}(y)\right)^{\perp} \subseteq \mathbf{R}^{m}$ в $\operatorname{Im} \sigma_{t}(y) \subseteq \mathbf{R}^{n}$. Определим оператор $\chi_{t}(y): \mathbf{R}^{n} \rightarrow \mathbf{R}^{m}$ следующим образом: $\chi_{t}(y)$ отображает $\operatorname{Im} \sigma_{t}(y)$ на $\left(\operatorname{ker} \sigma_{t}(y)\right)^{\perp}$ как обратный к $\sigma_{t}(y) ; \chi_{t}(y)$ равен нулю на $\left(\operatorname{Im} \sigma_{t}(y)\right)^{\perp}$. Очевидно, $\chi=\chi_{t}(y)-$ предсказуемый $\mathbf{R}^{m \times n}$-значный процесс на $C\left(\mathbf{R}_{+}, \mathbf{R}^{n}\right)$. Имеем $\chi_{t}(y) \sigma_{t}(y)=\varphi_{t}(y)$. Следовательно,

$$
\int_{0}^{t} \varphi_{s}(X) d B_{s}=\int_{0}^{t}\left(\chi_{s}(X) \sigma_{s}(X)\right) d B_{s}=\int_{0}^{t} \chi_{s}(X) d M_{s},
$$

где $M_{t}=\int_{0}^{t} \sigma_{s}(X) d B_{s}=X_{t}-x-\int_{0}^{t} b_{s}(X) d s$. Учитывая (3.7), получаем

$$
B_{t}=\int_{0}^{t} \varphi_{s}(X) d B_{s}+\int_{0}^{t} \psi_{s}(X) d B_{s}=\int_{0}^{t} \chi_{s}(X) d M_{s}+\int_{0}^{t} \psi_{s}(X) d V_{s} .
$$

Процесс $M$ является измеримым функционалом $X$, в то время как $V$ независим от $X$. Итак, (3.8) показывает, что распределение $\operatorname{Law}\left(X_{t}, B_{t} ; t \geqslant 0\right)$ одно и то же для всех решений $(X, B)$. 
Доказ ат ель с т в т е о ремы 3.2. Фиксируем $t \geqslant 0$. Пусть $(X, B)$ - сильное решение уравнения $(1.1)$ на $\left(\Omega, \mathscr{F},\left(\mathscr{F}_{t}\right), \mathbf{P}\right)$. Тогда существует $\mathscr{H}_{t}$-измеримое отображение $\theta: C\left(\mathbf{R}_{+}, \mathbf{R}^{m}\right) \rightarrow \mathbf{R}^{n}$ такое, что $X_{t}(\omega)=\theta(B(\omega))$ для $\mathbf{P}$-п.в. $\omega$. Пусть $\left(\mathbf{Q}_{\omega}\right)_{\omega \in \Omega}-$ условное распределение $X_{t}$ относительно $\mathscr{F}_{t}^{B}$. Тогда $\mathbf{Q}_{\omega}=\delta_{\theta(B(\omega))}$ для $\mathbf{P}$-п.в. $\omega$.

Пусть теперь $(\widetilde{X}, \widetilde{B})$ - решение уравнения $(1.1)$ на $\left(\widetilde{\Omega}, \widetilde{\mathscr{F}},\left(\widetilde{\mathscr{F}}_{t}\right), \widetilde{\mathbf{P}}\right)$. Обозначим через $\left(\widetilde{\mathbf{Q}}_{\widetilde{\omega}}\right)_{\widetilde{\omega} \in \widetilde{\Omega}}$ условное распределение $\widetilde{X}_{t}$ относительно $\mathscr{F}_{t} \widetilde{B}$. Поскольку $\operatorname{Law}(\widetilde{X}, \widetilde{B})=\operatorname{Law}(X, B)$, заключаем, что $\widetilde{\mathbf{Q}}_{\tilde{\omega}}=\delta_{\theta(\widetilde{B}(\widetilde{\omega}))}$ для $\widetilde{\mathbf{P}}$-п.в. $\widetilde{\omega}$. Следовательно, $\tilde{X}_{t}=\theta(\widetilde{B}(\widetilde{\omega}))$ для $\widetilde{\mathbf{P}}$-п.в. $\widetilde{\omega}$, и поэтому случайная величина $\widetilde{X}_{t}$ согласована с пополнением $\mathscr{F}_{t}^{\widetilde{B}}$. Это и есть требуемый результат.

4. Применения полученных результатов. Опишем сначала интерпретацию теоремы 3.1 в терминах мартингальных проблем. Пусть $x \in \mathbf{R}^{n}$. Пусть $b-$ предсказуемый процесс на $C\left(\mathbf{R}_{+}, \mathbf{R}^{n}\right)$, принимающий значения в $\mathbf{R}^{n}$. Пусть $a-$ предсказуемый процесс на $C\left(\mathbf{R}_{+}, \mathbf{R}^{n}\right)$, принимающий значения в пространстве $n \times n$-матриц.

О п р е д е л ен и е 4.1. Решением $n$-мерной мартингальной проблемы $(x, b, a)$ называется мера $\mathbf{Q}$ на $C\left(\mathbf{R}_{+}, \mathbf{R}^{n}\right)$ такая, что

i) $\mathbf{Q}\left\{Y_{0}=x\right\}=1$ ( $Y$ обозначает канонический процесс на $\left.C\left(\mathbf{R}_{+}, \mathbf{R}^{n}\right)\right)$;

ii) для любого $t \geqslant 0$

$$
\int_{0}^{t}\left(\sum_{i=1}^{n}\left|b_{s}^{i}(Y)\right|+\sum_{i=1}^{n} a^{i i}(Y)\right) d s<\infty \quad \text { Q-п.н.; }
$$

iii) для любого $i=1, \ldots, n$ процесс $M_{t}^{i}=Y_{t}^{i}-\int_{0}^{t} b_{s}^{i}(Y) d s$ является $\left(\mathscr{H}_{t}, \mathbf{Q}\right)$-локальным мартингалом $\left(\left(\mathscr{H}_{t}\right)\right.$ обозначает каноническую фильтрацию на $\left.C\left(\mathbf{R}_{+}, \mathbf{R}^{n}\right)\right)$, и $\left\langle M^{i}, M^{j}\right\rangle_{t}=\int_{0}^{t} a_{s}^{i j}(Y) d s$ для любых $i, j=1, \ldots, n$.

По поводу более подробного описания мартингальных проблем cM. [9].

Вернемся теперь к СДУ (1.1). Положим $a_{t}(y)=\sigma_{t}(y) \sigma_{t}^{*}(y)$. Если $(X, B)$ - решение уравнения $(1.1)$, то мера $\mathbf{Q}:=\operatorname{Law}\left(X_{t} ; t \geqslant 0\right)$ является решением мартингальной проблемы $(x, b, a)$. Обратно, если $\mathbf{Q}-$ peшение мартингальной проблемы $(x, b, a)$, то существует решение $(X, B)$ уравнения (1.1) такое, что $\operatorname{Law}\left(X_{t} ; t \geqslant 0\right)=\mathbf{Q}$. Слабая единственность для (1.1) эквивалентна единственности решения мартингальной проблемы $(x, b, a)$.

Теорему 3.1 можно теперь переформулировать следующим образом.

Теорема 4.1. Пусть $(x, b, a)-n$-мерная мартингальная проблема. Пусть $\sigma$ - предсказуемый $\mathbf{R}^{n \times m}-$ значный прочесс на $C\left(\mathbf{R}_{+}, \mathbf{R}^{n}\right)$ 
такой, что $\sigma_{t}(y) \sigma_{t}^{*}(y)=a_{t}(y)$. Тогда единственность решения мартингальной проблемы $(x, b, a)$ влечет единственность решения $(n+m)$-мерной мартингальной проблемь

$$
\left(\left(\begin{array}{l}
x \\
0
\end{array}\right),\left(\begin{array}{l}
b \\
0
\end{array}\right),\left(\begin{array}{cc}
a & \sigma \\
\sigma^{*} & I
\end{array}\right)\right) .
$$

Приведем еще одно применение полученных результатов. Для уравнения (1.1) каждое из следующих свойств:

слабое существование; сильное существование;

слабая единственность; сильная единственность,

может выполняться или нарушаться. Итак, всего существует $16\left(=2^{4}\right)$ возможных комбинаций. Некоторые из этих комбинаций не реализуются (например, если есть сильная единственность, то должна быть и слабая единственность). Используя примеры 2.1-2.4, а также предложение 1.2 и теорему 3.2, можно для любой из этих комбинаций или привести пример соответствующего СДУ, или доказать, что эта комбинация не реализуется. Оказывается, что существует лишь 5 реализуемых комбинаций (см. табл. 1).

\begin{tabular}{|c|c|c|c|l|}
\hline $\begin{array}{l}\text { слабое } \\
\text { сущест- } \\
\text { вование }\end{array}$ & $\begin{array}{l}\text { сильное } \\
\text { сущест- } \\
\text { вование }\end{array}$ & $\begin{array}{l}\text { слабая } \\
\text { единст- } \\
\text { венность }\end{array}$ & $\begin{array}{l}\text { сильная } \\
\text { единст- } \\
\text { венность }\end{array}$ & реализуется/не реализуется \\
\hline \hline- & - & - & - & не реализуется, очевидно \\
\hline- & - & - & + & не реализуется, очевидно \\
\hline- & - & + & - & не реализуется, очевидно \\
\hline- & - & + & + & реализуется, пример 2.1 \\
\hline- & + & - & - & не реализуется, очевидно \\
\hline- & + & - & + & не реализуется, очевидно \\
\hline- & + & + & - & не реализуется, очевидно \\
\hline- & + & + & + & не реализуется, очевидно \\
\hline+ & - & - & - & реализуется, пример 2.4 \\
\hline+ & - & - & + & не реализуется, диаграмма 1 \\
\hline+ & - & + & - & реализуется, пример 2.2 \\
\hline+ & - & + & + & не реализуется, диаграмма 1 \\
\hline+ & + & - & - & реализуется, пример 2.3 \\
\hline+ & + & - & + & не реализуется, диаграмма 1 \\
\hline+ & + & + & - & не реализуется, диаграмма 2 \\
\hline+ & + & + & + & реализуется, очевидно \\
\hline
\end{tabular}

Таблица 1. Комбинации различных типов существования и различных типов единственности. Например, комбинация « +-+- » в строке 11 означает, что мы интересуемся уравнением, для которого существует решение, не существует сильного решения, имеет место слабая единственность, но нет сильной единственности. Таблица показывает, что такое СДУ приведено в примере 2.2 
Эта статья была написана во время пребывания автора в университете Миннесоты. Автор благодарен Н. В. Крылову и Н. Джейну за интересные обсуждения и гостеприимство. Автор признателен Д. Струку за замечание о связи теоремы 3.1 с мартингальными проблемами. Автор выражает благодарность А. Н. Ширяеву за важные советы и предложения.

\section{СПИСОК ЛИТЕРАТУРЫ}

1. Cherny A.S. On the strong and weak solutions of stochastic differential equations governing Bessel processes. - Stochastics Stochastics Rep., 2000, v. 70, № 3-4, p. 213-219.

2. Engelbert H.-J. On the theorem of T. Yamada and S. Watanabe. - Stochastics Stochastics Rep., 1991, v. 36, p. 205-216.

3. Ито К., Макжин Г. Диффузионные процессы и их траектории. М.: Мир, 1968, $394 \mathrm{c}$.

4. Jacod J. Weak and strong solutions of stochastic differential equations. - Stochastics, 1980 , v. 3, p. 171-191.

5. Jacod J., Mémin J. Weak and strong solutions of stochastic differential equations: Existence and stability. - Lecture Notes in Math., 1981, v. 851, p. 169-212.

6. Жакод Ж., Ширяев А. Н. Предельные теоремы для случайных процессов. Т. $1,2$. М.: Физматлит, 1994, 544 с.; 368 с.

7. Липчер P.ШШ., Ширяев А.Н. Статистика случайных процессов. М.: Наука, 1974, $696 \mathrm{c}$.

8. Revuz D., Yor M. Continuous Martingales and Brownian Motion. I. BerlinHeidelberg: Springer-Verlag, 1994, 560 p.

9. Stroock D.W., Varadhan S. R.S. Diffusion processes with continuous coefficients. I, II. - Comm. Pure Appl. Math., 1969, v. 22, p. 345-400; p. 479-530.

10. Stroock D.W., Varadhan S. R. S. Multidimensional Diffusion Processes. I. BerlinHeidelberg: Springer-Verlag, 1979, $338 \mathrm{p}$.

11. Yamada T., Watanabe S. On the uniqueness of solutions of stochastic differential equations. - J. Math. Kyoto Univ, 1971, v. 11, p. 155-167.

12. Звонкин A.K., Крылов Н. В. О сильных решениях стохастических дифференциальных уравнений. - Труды школы-семинара по теории случайных процессов (Друскининкай, 1974), ч. 2, Вильнюс, 1975, с. 9-88.

Поступила в редакцию 18.V.2001 